\section{Y-chromosome microdeletions are not associated with SHOX haploinsufficiency}

Dear Sir,

Dr Chianese et al. (2013) expressed concerns about their results reported in ' $Y$-chromosome microdeletions are not associated with SHOX haploinsufficiency' and our earlier publication 'Aberrations in pseudoautosomal regions (PARs) found in infertile men with Y-chromosome microdeletions' (Jorgez et al., 20l I). We wish to respond to several of their statements regarding our work in hopes of clarifying our findings.

Deletions of $\mathrm{Yq}$ and isodicentric $\mathrm{Y}$-chromosomes are two relatively common structural abnormalities affecting male fertility. Deletions of distal Yq frequently include pseudoautosomal region 2 (PAR2) while the isodicentric- $Y$ is characterized by duplication of PARI and sex determining region of the $Y$-chromosome (SRY) and deletion of PAR2 (Lange et al., 2009). We evaluated 87 men with Y-chromosome microdeletions by array comparative genomic hybridization $(\mathrm{aCGH})$ and/or qPCR for PARs abnormalities. Thirteen men (I5\%) had an abnormal karyotype involving the Y-chromosome; they had PAR abnormalities. In addition, seven men with a normal karyotype also displayed PAR abnormalities. The Chianese et al.'s study included I I patients (6\%) with abnormal karyotypes, but PAR-copy number variations (CNVs) were reported in five patients. If the karyotype of the remaining six involved Y-chromosome aberrations, then PAR-CNVs should be evident. Also, if an isodicentric $Y$-chromosome is present, then $C N V$ s should be present in both PARs. Since PAR-CNVs were not reported, the sensitivity of the methods employed is a concern and may explain the difference in the results since the majority of their patients were analyzed by qPCR only.

Chianese et al. stated that their discrepancy with our data may be due to methodological issues although no specific problems were mentioned. Our study included a complete analysis of high density $X$ - and Y-chromosome aCGH performed in 25 men with non-obstructive azoospermia (NOA). We confirmed the $\mathrm{CNV}$ s detected by aCGH and identified CNVs using qPCR for at least three genes in each PAR and the SRY gene in the additional patients. Chianese et al. analyzed only SHOX gene CNVs in the majority of samples studied. Therefore, it is possible that PAR microdeletions or microduplications not encompassing SHOX were missed. Notably, one of our patients had a PARI duplication that did not encompass SHOX and this was confirmed by both aCGH and SHOX qPCR-analysis. A sub-set of NOA-men in our study with Y-microdeletion and normal karyotype $(5.4 \%, 4 / 74)$ had PARI deletions that included SHOX (height not available). Chianese et al. identified men with a normal karyotype (2/I77), normal height and SHOXCNVs (duplications) but at a lower frequency ( $1.1 \%)$. The normal height of these two individuals is not surprising as individuals with SHOX duplications have variable height.
Chianese et al. mentioned that our patients' phenotype was not fully described. Our paper states that patients were evaluated according to the American Urological Association Practice Guidelines (Gangel, 2002). Diagnosis was idiopathic NOA prior to Y-chromosome microdeletion testing. Our paper focused on SHOX aberrations as a co-existing genomic syndrome because height is an easily measured physical characteristic. Dosage changes of other PAR genes are associated with clear phenotypic anomalies, such as intellectual disability, but these characteristics may be difficult to ascertain. In our study, height was noted in the charts for only seven patients, with three men $<10$ th percentile and one male $>95$ th percentile (outside defined ranges of normalcy). One Y-isodicentric man displayed the typical body habitus and arm deformities characteristic of $\mathrm{SHOX}$ syndrome.

Over 200 individuals who were not infertile (C.J. Jorgez and D.J. Lamb, unpublished) and I 12 normospermic, but apparently infertile, controls by Chianese et al. did not exhibit CNVs in SHOX. SHOX-CNVs were present only in Y-chromosome microdeleted patients. The Database of Chromosomal Imbalance and Phenotype in Humans using Ensembl Resources (DECIPHER database) (https://decipher.sanger.ac.uk/) indicates that 35 of 29626 subjects $(0.11 \%)$ have SHOX CNVs. Of those patients with a phenotype described, $89 \%$ have intellectual disability. One DECIPER male with a PAR I-gain and Yq-deletion had azoospermia, as well as glaucoma and abnormalities of pyramidal motor function. Thus, SHOX CNVs rarely occur in the general population, which further supports the significance of identifying PAR-CNVs in Y-chromosome microdeleted patients.

In conclusion, we are pleased that the findings of Chianese et al., essentially confirm our earlier publication. Molecular studies using aCGH and CNV-qPCR of patients with Y-chromosome microdeletions allow the identification of additional, previously unrecognized $Y$ structural variations in NOA men. Additional Y-chromosome microdeletion men should be tested for PAR CNVs to define the incidence with certainty. Although the function of many PAR genes is under investigation, there may be consequences for male offspring of NOA men with PAR rearrangements who conceive using assisted reproductive technologies. Accordingly, further studies are needed to better understand the risks that may be present and this is an area where Chianese et al. and our laboratory certainly agree.

\section{References}

Chianese C, Lo Giacco D, Tuttelmann F, Ferlin A, Ntostis P, Vinci S, Balercia G, Ars E, Ruiz-Castane E, Giglio S et al. Y-chromosome microdeletions are not associated with SHOX haploinsufficiency. Human Reprod 2013;28:3155-3160.

Gangel EK, American Urological Association I and American Society for Reproductive M. AUA and ASRM produce recommendations for male infertility. American Urological Association, Inc. and American Society for Reproductive Medicine. Am Fam Phys 2002;65:2589-2590.

(C) The Author 2014. Published by Oxford University Press on behalf of the European Society of Human Reproduction and Embryology. All rights reserved.

For Permissions, please email: journals.permissions@oup.com 
Jorgez CJ, Weedin JW, Sahin A, Tannour-Louet M, Han S, Bournat JC, Mielnik A, Cheung SW, Nangia AK, Schlegel PN et al. Aberrations in pseudoautosomal regions (PARs) found in infertile men with Y-chromosome microdeletions. J Clin Endocrinol Metab 201I; 96:674-679.

Lange J, Skaletsky H, van Daalen SK, Embry SL, Korver CM, Brown LG, Oates RD, Silber S, Repping S, Page DC. Isodicentric $Y$ chromosomes and sex disorders as byproducts of homologous recombination that maintains palindromes. Cell 2009; I 38:855-869.

Carolina J.Jorgez ${ }^{1,2, *}$, John W. Weedin ${ }^{2}$, Aysegul Sahin ${ }^{2}$, Mounia Tannour-Louet ${ }^{2}$, Shuo $\mathrm{Han}^{3}$, Juan C. Bournat', Anna Mielnik ${ }^{4}$, Sau Wai Cheung ${ }^{5}$, Ajay Nangia ${ }^{6}$ Peter N. Schlegel ${ }^{4}$, Larry I. Lipshultz ${ }^{1,2}$ and Dolores J. Lamb ${ }^{1,2,3, *}$ 'Center for Reproductive Medicine, Houston, TX, USA

${ }^{2}$ Scott Department of Urology, Baylor College of Medicine, Houston, TX, USA

${ }^{3}$ Department of Molecular and Cell Biology, Baylor College of Medicine, Houston, TX, USA

${ }^{4}$ Department of Urology, Weill Medical College of Cornell University, New York, NY, USA ${ }^{5}$ Department of Molecular and Human Genetics, Baylor College of Medicine, Houston, TX, USA

${ }^{6}$ Department of Urology, The University of Kansas Medical Center, Kansas City, KS, USA

*Correspondence address. Center for Reproductive Medicine and Scott Department of Urology, Baylor College of Medicine, Houston, TX77030, USA. Tel: + I-7| 3-798-354I; E-mail: cj I29804@bcm.edu (C.J.J.); Center for Reproductive Medicine and Scott Department of Urology, Baylor College of Medicine, Houston, TX 77030, USA. Tel: + I-7I3-798-7266; E-mail: dlamb@bcm.edu (D.J.L.)

doi: 10.1093/humrep/deu038 Advanced Access publication on March I4, 2014

\section{Reply: Y-chromosome microdeletions are not associated with SHOX haploinsufficiency}

\section{Dear Sir,}

This letter addresses the debate between our recently published paper (Chianese et al., 20I3) and the earlier publication of Jorgez et al. (20I I). Overall, we feel that Jorgez et al. missed the main objective of our study, which was SHOX gene dosage and not pseudoautosomal region-I (PARI)-related copy number variations (CNVs). We investigated this specific aspect, as the alarming finding of SHOX haploinsufficiency in $5.4 \%$ of azoospermia factor (AZF) deletion carriers with normal karyotype (Jorgez et al., 20 I I) raised much concern for the genetic counselling of $Y$-chromosome deletion carriers. Our conclusion was completely different and analytical issues have been proposed to explain the differences.

Firstly, Jorgez et al. opine about the sensitivity of our methods because in 6 of II patients with abnormal karyotype we did not find any PARrelated CNVs. We are fully confident about the sensitivity of the methods employed, since the type of karyotype anomaly could explain the lack of CNVs in these patients. Compatible with our finding, one of these patients had a mosaic karyotype: 46, X, t (Y;|6)(q 2;q24)[30]/ $46, X Y[70]$; the analysis by both array comparative genomic hydridisation $(\mathrm{aCGH})$ and $\mathrm{qPCR}$, gave coherent results: i.e. the lack of PAR-linked CNVs and unaltered SHOX dosage, respectively. Karyotypes of the remaining five patients also involved $\mathrm{Y}$-chromosome aberrations, but SHOX CNVs were not found because: (i) mosaic karyotype: 45,X[10]/46,X,idic(Y)(q I I.22) [40]; and (ii) 46,XX male (SRY positive).

Furthermore, in their Letter to the Editor Jorgez et al. claim that 'if an isodicentric $Y$-chromosome is present, then $C N V$ s should be present in both PARs', probably referring to our patient A416, for whom we only reported a gain in PARI detected by a-CGH (Table I in our original paper). We are glad they mentioned this case, because in fact raw data indicated that this patient had a gain in PARI (Xp22.33 and YpII.32-pII-3I; log score $=0.617)$ and a loss in PAR2 (Xq28 and Yql I.222-qI2; log score $=-0.627$ ).

Jorgez et al. questioned our choice of analyzing only the SHOX gene. As stated above, we believe that the most striking finding reported by the previous publication was the identification of a sub-set of men with non-obstructive azoospermia (NOA) with $\mathrm{Y}$-microdeletion and normal karyotype $(4 / 74 ; 5.4 \%)$ displaying PARI deletions including SHOX, i.e. SHOX haploinsufficiency. For instance, the aim of our study was to prove whether, in our larger (almost double) study population, Y-chromosome microdeletions were associated with SHOX haploinsufficiency: to accomplish this, testing for other PAR genes was unnecessary.

The point made by Jorgez et al. concerning SHOX duplications and individuals' height, is unclear to us. We do state in the discussion 'Literature is poor and unclear about SHOX over-dosage, which has been reported in association with normal to tall stature [...]'. We actually find it strange that in their publication, information on height is not reported for the four patients with normal karyotype carrying SHOX deletions. In general, if SHOX deletions are present then short stature and/or skeletal abnormalities should be present as well, but this is not reported by Jorgez et al.

We are sorry but we do not agree with the statement: ' $[. .$.$] the find-$ ings of Chianese et al. (20I3) essentially confirm our earlier publication'. Although we did find SHOX duplications in I. I\% of men with Y microdeletions and normal karyotype, it does not mean that this category of patients is at higher risk for SHOX CNVs compared with the general population. The reference that Jorgez et al. make to the DECIPHER database (Database of Chromosomal Imbalance and Phenotype in Humans using Ensembl Resources), reporting only $0.11 \%$ of SHOX CNVs, is misleading. This database is mainly based on arrays with a relatively low resolution (probes every $0.5-1 \mathrm{Mb}$ ) that may not allow the detection of small rearrangements such as ours. Moreover, all CNVs involving SHOX in the DECIPHER database had a huge size (the majority ranging from 5-58 Mb). It is worth noting that the pathologic phenotype of the azoospermic man with Yq microdeletion and a duplication of I I.6 Mb mentioned in the letter by Jorgez et al. is not an appropriate example to demonstrate an association between $\mathrm{Yq}_{\mathrm{q}}$ deletions and PARI linked duplications. For instance, this large duplication extends over PARI and clearly affects the $X$ chromosome, duplicating a total of 43 genes.

The only point we agree on with Jorgez et al. is the need for further studies concerning SHOX duplications; in fact, we considered the low number of analyzed controls a limitation to prove that these $C N V s$ are actually associated with the presence of microdeletions.

In conclusion, we provided the largest collection of men carrying Y-chromosome microdeletions analyzed so far in association with SHOX CNV. Our data show no association between Y-chromosome microdeletions and SHOX haploinsufficiency in men with normal karyotype, which is in net discordance with the previous findings of $5.4 \%$ of NOA-men with Y-microdeletion and normal karyotype. 